\title{
Optimized Wireless Stethoscope Using Butterworth Filter
}

\author{
Ms. Mohini Dadhe, Ms. Sneha Miskin \\ Research Scholar, Department of Computer Engineering \\ B.D.C.E Sevagram Maharashtra India \\ snehamiskin@gmail.com
}

\begin{tabular}{|c|c|}
\hline Article History & Abstract \\
\hline $\begin{array}{l}\text { Article Submission } \\
10 \text { May } 2015 \\
\text { Revised Submission } \\
27 \text { July } 2015 \\
\text { Article Accepted } \\
10 \text { August } 2015 \\
\text { Article Published } \\
30 \text { September } 2015\end{array}$ & $\begin{array}{l}\text { Acoustic stethoscope is a primary diagnostic tool used by doctors for effective } \\
\text { analysis of various diseases. But, it suffers defects such as low level heart sounds and } \\
\text { presence of murmurs. This project aims at developing 'Noise removal in stethoscope } \\
\text { using Butterworth filter'. In this, the low level heart sounds were amplified with high } \\
\text { gain pre-amplifier circuit. A review of basic filters like low pass RC filter, } \\
\text { Butterworth filter, Chebyshev filter and Bessel filter are done for elimination of } \\
\text { murmurs and best suited filter is suggested. Finally, amplified noise free heart sounds } \\
\text { are simulated using Proteus 7.4. The results prove that efficient filter for designing is } \\
\text { 'Butterworth filter'. } \\
\text { Keywords: Acoustic stethoscope, Heart murmurs, Sallen-Key Buterworth low-pass } \\
\text { Filter, Power amplifier }\end{array}$ \\
\hline
\end{tabular}

\section{Introduction}

Auscultation refers to the listening of heart sound in human body and the tool used for such purpose is known as a stethoscope. Traditional stethoscopes use mechanical means for auscultation resulting in poor sound quality and low accuracy as the signal can get affected by the listening capability of the doctor as well as by the unfavorable environment. This paper proposes the design of low cost affordable wireless stethoscope based on $\mathrm{RF}$ technology to process the heart beat signal, which can transmit and stores the signal for wireless electronic stethoscopes are based on Zigbee and Bluetooth technology which makes them a better choice for implementation of wireless stethoscope but keeping in mind the cost incurred for developing such systems we have to think for the alternatives to make the system affordable with a trade-off in terms of quality and cost, however Zigbee and Bluetooth provides better sound quality but this problem is almost eliminated in RF based wireless stethoscope by using pre-amplifier, filter and power amplifier with variable gains to achieve the noise free results as proposed by Zigbee and Bluetooth [6][7].

\section{Related Work}

Rene Laennec, a French physician invented the first ever stethoscope in 1816. First of their kinds were comprised of wooden tubes having trumpet-like ends, since then this particular tool saw many improvements in itself such as binaural constructions, flexible tubes and interactive designs but the basic functionality and use of mechanical means for listening the heart sound remained unchanged for almost one and a half century. Chien et al. in 2004 proposed an idea for wirelessly transmitting the heart sounds by using Bluetooth as a transmitting medium at both ends to display and store in a PDA as a phonocardiograph data.

Jatupaiboon et al. in 2010 proposed a prototype for storing the heart beat sound filtering it by means of low pass filters, the purpose of this paper was focused on reducing the noise from the said data (Tang et al. in 2010), proposed a wireless electronic stethoscope by using Bluetooth in integration with embedded processors for displaying the data on LCD and storing the data for computer-aided diagnosis. (Harsola et al. 2011) proposed a design of Peripheral Interface Controller based electronic stethoscope for visualizing and storing of sound data 
suggesting that the stethoscope should be physically connected and data will be sent through Lab View[5]. (Gururajan et al. 2011) conducted a study based on different de-signs of wireless electronic stethoscopes and their feasibility based on user's perspective to identify issues when using these stethoscopes in telemedicine and e-health platforms [6]. (Patil, et al. 2012) proposed the design of contact vibration sensor to minimize the ambient noise in acquiring the heart sound signal, for wireless transmission the authors used Bluetooth for ehealthcare implementation [2].

(Wang et-al ,2009)proposed the heart sound measurement and analysis system with digital stethoscope, this paper is concerned with the cardiac sound measurement and analysis system for in-home use of heart abnormality monitoring[8][9]. (Yashaswini et-al 2012) reviewed the design of electronic stethoscope, this paper reviews efficiency \& cost-effective design of power supply, sensor, pre-amplifier, low pass filter \& power amplifier[4][10].

\section{Proposed Wireless Stethoscope Using Butterworth Filter}

Heart sounds are referred to as acoustic waves generated by the resultant flow of blood and heartbeat. These vibrations are generated by opening and closing of systole/diastole valves. Heart sound is of two type waves i.e. S1 wave (Systole) and S2 wave (Diastole). Sometimes the valve doesn't work normally, either the valve doesn't opens properly which causes less blood flow or the valve doesn't close tightly causing blood leak backwards, these abnormalities are referred to as murmurs. Murmurs can be systolic or diastolic as shown in (Fig. 2).

Murmurs and heartbeats have band limit frequencies between $100-1000 \mathrm{~Hz}$ and have a relative low intensity that makes the human hearing perceptible to heart sound signal, therefore the auscultation using acoustic stethoscopes seems to be a bit difficult.

Keeping in view that the traditional stethoscopes have two sides for observing internal sounds, the bell and the diaphragm, different frequencies are used for diagnosis. One uses $20-500 \mathrm{~Hz}$ range for hearing heart sound and pumping of blood vessels and other uses $200-1000 \mathrm{~Hz}$ frequency range to listening respiratory signals 1 and 5 . Considering the system architecture of traditional method a low pass filter is designed to eliminate the frequencies above $1000 \mathrm{~Hz}$ for reducing the noise, hence picking up sounds having low frequencies. Heart sounds are picked up by chest piece of mechanical stethoscope. It is then amplified with high gain pre-amplifier. Murmurs are eliminated using 'Low pass Butterworth filter' \& wirelessly transmitted using RF module. Power amplifier is used for driving signals which could be heard by headphones or stored in MP3 player for further analysis [2].

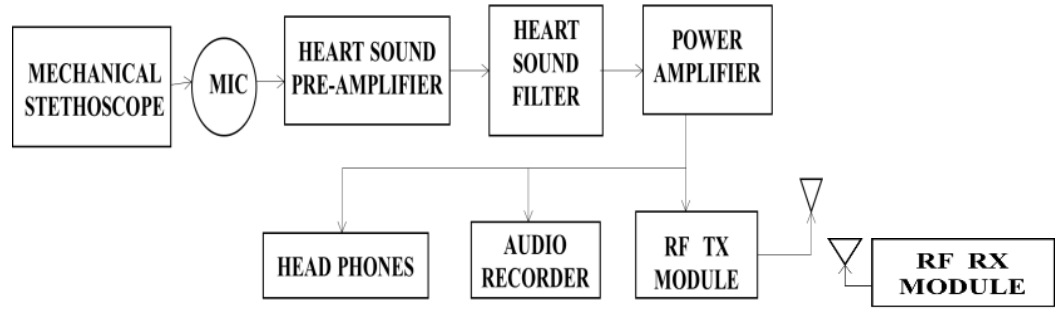

Fig 1: Schematic representation of transmitter and receiver

\section{A) Preamplifier}

An OP-AMP (Operational Amplifier) is used in pre-amplifier section for achieving a voltage gain of 3.For achieving this, LM358 OP-AMP was chosen due to its low power consumption which draws a small amount of current and can be powered by a 3-volt DC supply, another reason for choosing LM358 OP-AMP is its low cost which helps us achieve two of three proposed objectives. 


\section{B) Filter}

In order to minimize the murmurs occurring in heart sounds, analog low pass filters such as Low pass RC filter, Chebyshev filter, Bessel filters and Butterworth filter are designed and simulated using Proteus 7.4. Chebyshev filter, Bessel filters and Butterworth filter are designed using Sallen Key topology.

\section{C) Low-pass RC filter}

Low-pass filters provide a smoother form of a signal, removing the short-term fluctuations, and leaving the longer-term trend .Low-pass RC filter can be used for limiting the audible frequencies. The cut-off frequency for this particular filter is set to be $500 \mathrm{~Hz}$, which means that the frequencies below this cut-off frequencies will be passed and frequencies above the cut-off frequency will be left out.

\section{Simulation Results}

Chebyshev low pass filters are analog or digital filters having a steeper roll-off and more pass band ripple (type I) or stop band ripple (type II) than Butterworth filters. Chebyshev filters have the property that they minimize the error between the idealized and the actual filter characteristic over the range of the filter, but with ripples in the pass band. Because of the pass band ripple inherent in Chebyshev filters, the ones that have a smoother response in the pass band but a more irregular response in the stop band are preferred for some applications. The time-domain step response of the Bessel filter has some overshoot, but less than common frequency domain filters. The proposed hardware is shown in figure 2 .

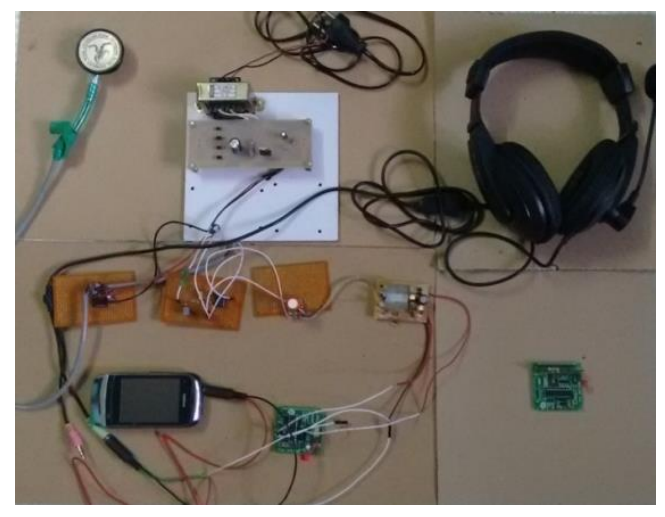

Fig 2: Wireless Stethoscope Using Butterworth Filter

The Butterworth low pass filter is a type of signal processing filter designed to have as flat a frequency response as possible in the pass band. It is also referred to as a maximally flat magnitude filter. The frequency response of the Butterworth filter is maximally flat (i.e. has no ripples) in the pass band and rolls off towards zero in the stop band. Finally, simulated results in Proteus 7.4 proves that murmur is eliminated to a great extent in this filter. So, Butterworth LPF is used for design.

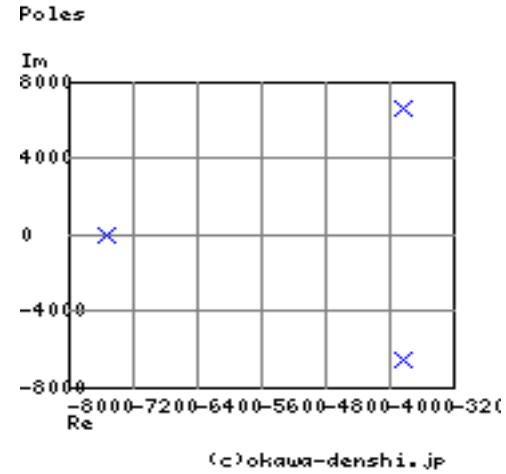

Fig 3: Poles plot of Butterworth LPF 
Due to low cost RF modules used for transmitting and receiving heart signals .RF transmitter and receiver helps doctors in diagnosing signals irrespective of location and distance. This module also helps in analysis of heart sounds by more doctors [1]. An audio power amplifier is an electronic amplifier that amplifies low-power audio signals (heart sounds composed primarily of frequencies between $20-200 \mathrm{~Hz}$,) to a level suitable for driving headphones. Amplification is achieved by using LM386 which is a power amplifier and can achieve the gain up to 40. Heart sounds can be continuously recorded. This can be achieved by connecting condenser microphones to headphones which are further connected to MP3 player, storing heart sounds.MP3 player can be interfaced with PC for further analysis [5].

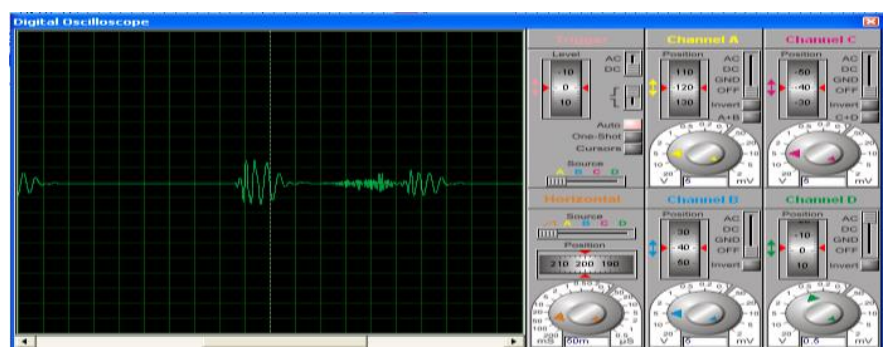

Fig 4: Late systolic murmur input

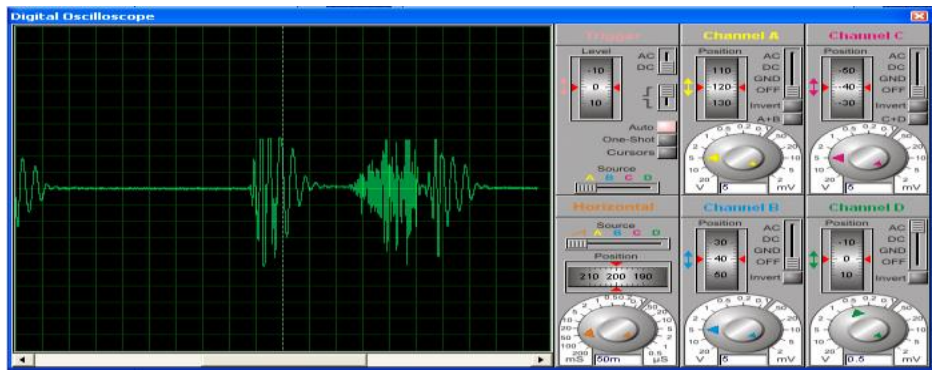

Fig 5: Bessel and pre-amplifier output

Design of pre-amplifier, analog filters such as Low pass RC filter, Chebyshev LPF, Bessel LPF, Butterworth LPF are simulated using Proteus 7.4 and following results are obtained. The designed architecture of entire circuit with pre-amplifier, Butterworth LPF and power amplifier are implemented in hardware.

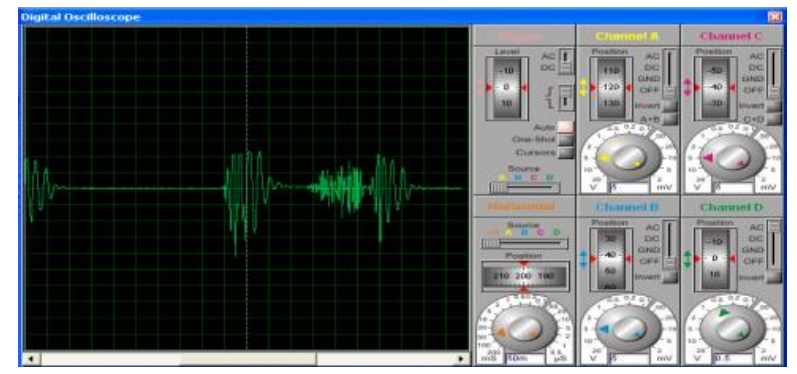

Fig 4: Butterworth output

\section{Conclusion}

A design of low cost electronic wireless stethoscope has been proposed and analysis based on simulations has been carried out in this paper. The proposed system have the functionality to store the data for further analysis and effective diagnosis hence improving the accuracy in diagnosis of cardio vascular diseases. The hardware design can be implemented in future with additional functionalities such as provision for viewing heard sounds in GLCD. This system will help doctors in accurate diagnosis and analysis of heart sounds. 


\section{References}

[1] Mulhasan, S. A. Khowaja, Z. Qazi, a. A. Shah,(2014). "A cost-effective indigenous wireless electronic stethoscope using RF” Sindh Univ. Res. Jour. (Sci. Ser.) Vol.46 (4):499-504 (2014).

[2] Patil, D. D. K., and R. K. Shastri, (2012). "Design of Wireless Electronic stethoscope based on zigbee" Inter-national Journal of Distributed and Parallel Systems (IJDPS) Vol.3, No.1. 351-359.

[3] Wang, H., J. Chen, and C. Samjin, (2009) "Heart Sound Measurement and Analysis System with Digital Stetho-scope", International Conference on Biomedical Engineering and Informatics.

[4] Yashaswini, B.S., and B.S. Satyanarayana, (2012). "Design of an Electronic Stethoscope: Review", Interna-tional Conference on Computer Science and Information, 37-41.

[5] Harsola, A., S. Thale, and M. S. Panse, (2011) "Digital Stethoscope for Heart Sounds", International Conference and Workshop on Emerging Trends and Technologies, 42-45.

[6] Gururajan, R., H. S. Tsai, and H. Chen, (2011) "Using Digital Sthethoscopes in Remote Patient Assessment via Wireless Networks: The User's Perspective" International Journal of Advanced Networking and Applications, Vol. 3, No. 3, 1140-1146.

[7] W. Y. Shi, J. Mays and J. -. Chiao, "A wireless stethoscope," 2015 IEEE MTT-S 2015 International Microwave Workshop Series on RF and Wireless Technologies for Biomedical and Healthcare Applications (IMWS-BIO), Taipei, 2015, pp. 197-198, doi: 10.1109/IMWS-BIO.2015.7303845.

[8] E. Türk et al., "Wireless bioacoustics sensor system for automatic detection of bowel sounds," 2015 19th National Biomedical Engineering Meeting (BIYOMUT), Istanbul, 2015, pp. 1-4, doi: 10.1109/BIYOMUT.2015.7369458.

[9] Y. He and S. Lian, "Stethoscope: A Sustainable Runtime Debugger for Wireless Sensor Networks," 2015 IEEE 21st International Conference on Parallel and Distributed Systems (ICPADS), Melbourne, VIC, 2015, pp. 250-257, doi: 10.1109/ICPADS.2015.39.

[10] J. Jusak and I. Puspasari, "Wireless teleauscultation for phonocardiograph signal recording through Zigbee networks," 2015 IEEE Asia Pacific Conference on Wireless and Mobile (APWiMob), Bandung, 2015, pp. 95-100, doi: 10.1109/APWiMob.2015.7374939. 